\title{
Serological diagnosis of HIV infection: Practice and performance in western Europe
}

\author{
A A Glynn
}

\begin{abstract}
Current laboratory practice in testing for HIV antibodies in western Europe was investigated by means of a questionnaire addressed to the 12 EC countries and Finland, Norway, Sweden and Switzerland. Despite inevitable regional differences there was a fair degree of homogeneity in broad laboratory organisation and in the types of tests and confirmatory strategies used. The primary test is always some form of enzyme linked immunosorbent assay (ELISA), though a number of laboratories also use agglutination tests. Confirmation is by an ELISA of a different type, or by Western blot, or both. The size and workload of laboratories covered a wide range. It is suggested that laboratories doing only a small number of tests at infrequent intervals should take extra care in validating their results and should be closely monitored. Twelve of the 16 countries studied have a quality assurance scheme for monitoring laboratory performance. Participation is voluntary but is invaluable even for the largest laboratories.

The results suggest that the standard of laboratory diagnosis is reasonably uniform throughout the region, which is not only important for the individual patient but means that epidemiological comparisons of data from different areas have at least a sound technical base.
\end{abstract}

The most reliable method of diagnosing an infection is to isolate the causal organism. Esoteric arguments on the interpretation of $\mathrm{H}$ or $\mathrm{O}$ antibody titres in the diagnosis of typhoid fever therefore became irrelevant as methods of isolation of Salmonella typhi improved. Where culture is impossible antibodies may be diagnostically invaluable, as in syphilis, or useless, as in leprosy. Although techniques have greatly improved since the human immunodeficiency virus (HIV) was first discovered, virus isolation is not yet a routine method of diagnosis. In contrast, the detection of antibodies to HIV is widely practised and is more efficient than many of the serological tests traditionally accepted in medicine.

Because a positive result has far reaching consequences for the individual patient, it is generally accepted that it should only be reported if confirmed by at least two tests, preferably based on different principles. Moreover, those tests best for individual diagnosis are not necessarily the most suitable for screening, such as those used for blood donors, or for epidemiological surveillance. Until recently the latter has largely been based on reports of patients with AIDS-that is, in whom infection occurred several years earlier. Epidemiological study of HIV now depends more and more on serological data, whether obtained in routine diagnosis or by special surveys.

To deal with these problems codes of practice have grown up in centres where many tests are done. Such codes may differ from country to country and between individual laboratories in the same country. To ensure adequate levels of diagnosis and comparability of epidemiological data, some degree of common standardisation of reagents and practice would be desirable.

As a first step, an attempt was made to get information on current laboratory practice in western Europe by means of a questionnaire addressed to the 12 countries of the European Community, together with Finland, Norway, Sweden and Switzerland.

\section{Methods}

The questionnaire had three sections dealing with general organisation, primary laboratories, and confirmatory laboratories. The appropriate central authority in each country was asked to complete the general section and to distribute the others to six primary and six confirmatory laboratories. They were asked to choose laboratories representative of all kinds, large and small, but were not given any more detailed requirements or guidance on selection methods.

The questions were designed to give information on the numbers, size, and workloads of laboratories, the types of tests used, and the underlying strategies. Information was also sought on the presence and extent of quality assurance schemes for laboratory performance. The quality control of individual test methods or kits was not part of the inquiry.

\section{Results}

Because of the rather free method of selection the laboratories questioned are not a random sample and should not be regarded as a truly representative one. Moreover, 12 laboratories are proportionately a much smaller sample from say, France, than from Finland. In fact 
Table 1 Size of populations served by primary laboratories

\begin{tabular}{lc}
\hline Country & $\begin{array}{c}\text { Population } \\
\text { per 1000/primary laboratory }\end{array}$ \\
\hline Belgium & $13 \cdot 5$ \\
Denmark & 255 \\
Eire & 292 \\
Finland & 357 \\
France & 23 \\
Germany & 300 \\
Greece & $?$ \\
Italy & 163 \\
Luxembourg & 200 \\
The Netherlands & 233 \\
Norway & 221 \\
Portugal & 102 \\
Spain & $?$ \\
Sweden & 237 \\
Switzerland & 165 \\
United Kingdom & 204 \\
\hline
\end{tabular}

more than 12 replies were received from some countries, but this was counterbalanced by a deficit from others. Nevertheless, there is a sufficiently common pattern among the 100 primary and 70 reference laboratories who replied, to be of some interest.

\section{GENERAL ORGANISATION}

In most countries there are both primary and confirmatory testing laboratories, although the distinction is not always recognised in the formal organisation. In England and Wales there are eight recognised confirmatory laboratories, including the Central Public Health Laboratory (CPHL) at Colindale which acts as a final court of appeal for the United Kingdom. There are also reference laboratories in Scotland, Northern Ireland, and Eire. Denmark, Finland, Norway, Portugal, Spain and Sweden all have national central reference laboratories for HIV tests based on a pre-existing central laboratory for clinical pathology. Neither France nor the former German Federal Republic recognise special reference laboratories for HIV tests, but in practice some of their larger regional or university laboratories behave as such. In Italy the central government recommends primary laboratories, with a relatively small number of confirming laboratories, but the 20 regions differ in organisation.

Whether a clinical laboratory tests for HIV antibody depends largely on local demand and resources, but is also affected by the way

Figure 1 Workload (tests/month) in diagnostic, blood transfusion, and mixed laboratories.

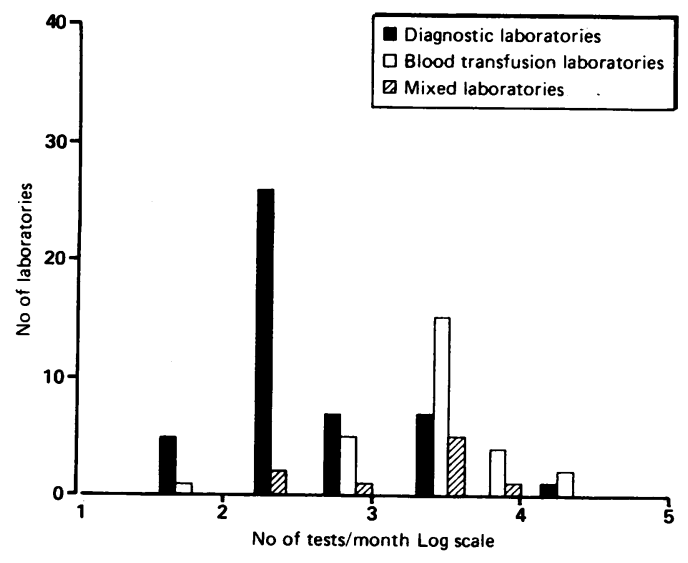

Table 2 Types of test used in 100 primary laboratories

\begin{tabular}{lc}
\hline Test & No of laboratories \\
\hline Abbot Recombinant & 50 \\
Abbot Envacore & 8 \\
Wellcozyme Recombinant & 32 \\
Pasteur Elavia & 22 \\
Pasteur LavBlot & 3 \\
Pasteur Peptilav & 3 \\
Organon Vironostika & 15 \\
Behring Ezygnost & 12 \\
Other ELISA & 34 \\
Fujirebo Serodia & 13 \\
Western Blots & 8 \\
Others & 13 \\
Total & 213 \\
\hline
\end{tabular}

clinical pathology in general is organised and by government policy. For example, in England and Wales testing was at first largely confined to 12 Public Health and 12 Blood Transfusion Service laboratories, together with some university and research laboratories. Any recognised laboratory may test if it so wishes and over 200 now do.

It looks as if in western Europe generally there is one primary HIV laboratory for 150 300000 people whatever the prevalence of AIDS in the country (table 1). The two exceptions to this are Belgium and France where the figures are 14000 and 23000 , respectively. This may be due to the involvement of a large number of private laboratories.

\section{PRIMARY LABORATORIES}

The workload in the 100 primary clinical laboratories analysed ranged from under 100 to over 1000 tests a month (fig 1). While most blood transfusion laboratories do several thousand tests a month and two do over 10000 , they see relatively few positive sera.

Forty three per cent of primary laboratories use only one type of test and refer positive or equivocal sera elsewhere. Thirty four per cent use two tests and the rest anything up to seven. All laboratories use some type of ELISA and some use an agglutination test. Few do Western blots (table 2).

\section{CONFIRMATORY LABORATORIES}

Of the 67 confirmatory laboratories who answered the questionnaire, eight were central national laboratories; the rest deal only with regional or local specimens. Thus most confirmatory laboratories deal with less than 20 primary ones while four only deal with their own specimens (fig 2). Four laboratories do over 200 confirmatory tests a month while 45 do less than 50 , and five, only 10 or less (fig 2 ).

Most laboratories test daily or at least weekly, but six only test at intervals of a month or more.

The most common strategy among confirmatory laboratories is to repeat the original test together with another type of ELISA and to follow this with a Western blot. Only 52 out of the 67 laboratories test a second sample of serum, essential if errors of sample identification are to be avoided. There are, however, many strategic variations (table 3 ). Where necessary the three laboratories who never use 
Figure 2 Workload in confirmatory laboratories: (A) number of tests a month; (B) number of laboratories serviced.

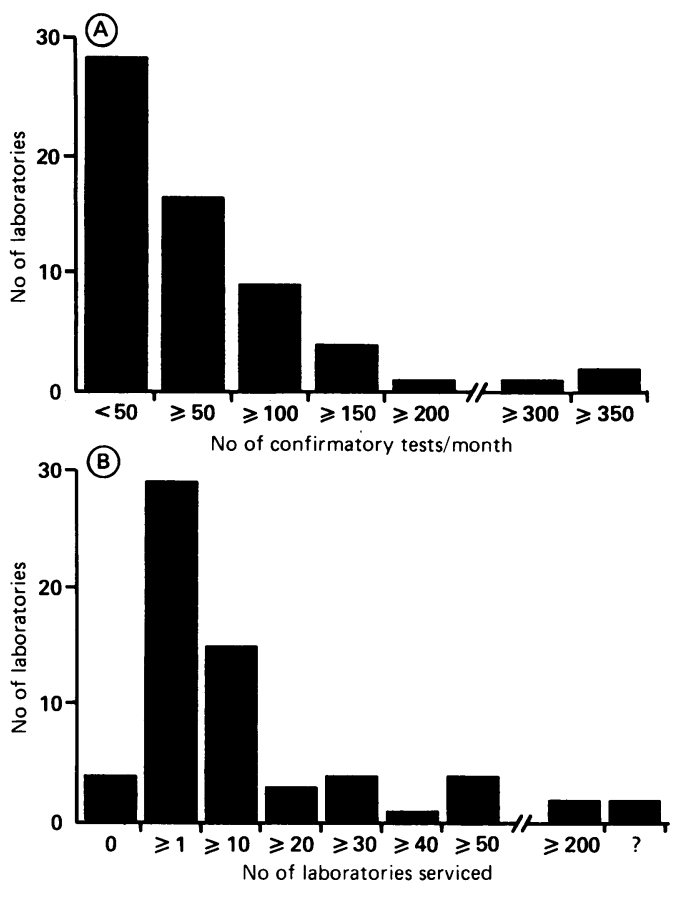

Western blots refer difficult sera to a national reference laboratory. The four laboratories who use nothing but Western blots are national or very large reference or blood transfusion laboratories. A few laboratories also use a radioimmunoprecipitation or other radioimmunoassay, immunoblots, or, in one, Serodia's agglutination test. Thirty four laboratories use the DuPont Western blot system, eight the Pasteur, and five both. Five use in-house systems.

\section{HIV-2 ANTIBODY TESTS}

The detection of HIV-2 antibodies is becoming increasingly important although 40 per cent of primary and 15 per cent of confirmatory laboratories still do not look for them (table 4). In the United Kingdom where extremely few cases have been detected, all blood donors are being screened from June 1990. Previously only those with West African connections were tested specifically for HIV-2. Anti-HIV-1 ELISAs often detect HIV-2 but this cannot
Table 5 Quality assessment schemes

\begin{tabular}{lcl}
\hline Country & $\begin{array}{c}\text { Central } \\
\text { scheme }\end{array}$ & Others used \\
\hline Belgium & + & \\
Denmark & + & CPHL \\
Eire & + & \\
Finland & + & Scientific Societies \\
Germany & 0 & \\
Greece & 0 & \\
Italy & + & Belgian \\
Luxembourg & 0 & \\
The Netherlands & + & \\
Norway & + & Centres for Disease Control \\
Portugal & 0 & \\
Spain & 0 & \\
Sweden & + & Swiss Red Cross, CPHL, \\
Switzerland & + & Centres for Disease Control \\
United Kingdom & + & \\
\hline
\end{tabular}

Abbreviations: CPHL, Central Public Health Laboratory, Colindale, England.

always be relied on, and if HIV-2 is suspected, or needs to be eliminated, a specific anti-HIV-2 or a combined test should be used. Laboratories vary in their use of such ELISAs (table 4) but more are changing to a combined test. The same applies to Western blots which may be specific for HIV-1 or HIV-2. Portugal, thanks to its historical connections with West Africa, has a far higher prevalence of HIV-2 than the rest of Europe. It now uses a combined ELISA and confirms positive results by means of both types of Western blot.

\section{QUALITY ASSURANCE}

Schemes in which test specimens are sent at regular intervals to laboratories which are assessed on their results are a valuable means of monitoring and improving laboratory performance. Where such schemes were already in operation for clinical pathology in general, HIV antibody tests have been added on. Otherwise, specific HIV schemes have been introduced except in four countries (table 5). Some countries also make use of the schemes available from the CPHL and the Control Centers for Disease, Atlanta. All schemes monitor ELISA results. In France they also send out test samples for Western blots three times a year.

\section{Discussion}

In all the countries questioned there is a system of primary and confirmatory laboratories even if in one or two they are not officially recognised as such. Although there are differences in detail, some form of ELISA is universal as the primary test, while Western blots are the predominant, though by no means the only, confirmatory test. When this survey was being planned the underlying question was to see how far organisation, methods, and results varied from country to country and whether more coordination and standardisation were indicated. The results of the questionnaire and related matters were discussed by virologists from the countries involved at a recent meeting. ${ }^{1}$ The view was that with the tests now available the overall quality of testing was high and formal common standards were not essen-
Included in HIV-1 tests

Tested for specifically but not in all sera Not tested for at all

Total

45
40
15
100


tial. Attempts to impose them would be difficult, particularly with the plethora of new tests appearing on the market.

The apparent uniformity is largely the effect of an international diagnostic reagent industry the quality of whose products is monitored in several countries and by the World Health Organisation. However good the reagents the validity of the results also depends on laboratory performance. The quality assurance schemes which many, but not all, countries run, not only monitor this performance but by doing so encourage its improvement. The rapid development of HIV antibody tests and their importance make the provision of quality assurance schemes perhaps even more desirable than of laboratory tests in general. There is a case for increased collaboration between the bodies running such schemes, though a centralised European scheme is not practicable.
Even with efficient staff and reliable methods, the results from a laboratory infrequently carrying out a small number of tests, must always be open to question. Such a practice may sometimes be convenient but independent confirmation is even more important than usual. Similar arguments apply even more strongly to reference laboratories occasionally doing small numbers of tests. As well as questions of validity it must mean that some patients have to wait too long before learning the results.

I thank the European Commission for a grant supporting this work, the members of the EC AIDS Research Working Party for advice, and PP Mortimer, JH Parry, and K Newton for advice and help.

1 Glynn AA. European meeting on HIV testing. $\mathrm{Br} \mathrm{Med} \mathrm{J}$ 1990;301:778. 